\title{
Fatores Ambientais e Genéticos sobre o Crescimento ao Ano e ao Sobreano de Bovinos Nelore, Criados no Nordeste do Brasil ${ }^{1}$
}

\author{
Stefano Biffani ${ }^{2}$, Raimundo Martins Filho $^{3}$, Alessandro Giorgetti ${ }^{4}$, Riccardo Bozzi ${ }^{5}$, \\ Francisco de Assis Melo Lima ${ }^{3}$
}

\begin{abstract}
RESUMO - Os dados de crescimento relativos a 2004 animais da raça zebuína Nelore, criados nos Estados do Ceará e do Piauí, foram usados para estudar a influência de fatores ambientais e estimar a herdabilidade das características peso aos 365 (ano) e 550 dias (sobreano) de idade. Os componentes de variância foram estimados pelo método da Máxima Verossimilhança Restrita. Na análise da variância foi usado um modelo que incluiu os efeitos fixos de sexo, ano, estação e rebanho de nascimento, a idade da mãe como covariável e o efeito aleatório de touro dentro de rebanho. Os efeitos fixos foram significativos para todas as características avaliadas. As médias ajustadas para esses efeitos foram: $186,16 \pm 2,74 \mathrm{~kg}$ e 244,06 $\pm 5,23 \mathrm{~kg}$, para os pesos aos 365 e 550 dias de idade, respectivamente. A idade da mãe ao parto influenciou somente o peso aos 365 dias, com peso máximo de 187,29 kg e idade em torno de nove anos. As estimativas de herdabilidade foram iguais a $0,56 \pm 0,09$ e $0,64 \pm 0,12$ para os pesos aos 365 e 550 dias, respectivamente.
\end{abstract}

Palavras-chave: peso corporal, herdabilidade, correlação genética

\section{Environmental and Genetic Effects on Yearling and Post-yearling Weights of Nellore Calves in Northeast Brazil}

\begin{abstract}
Data growth records relative to 2004 Nellore breed calves, from Ceará and Piauí states, were used to study the influence of environmental effects and to estimate the heritability of the weight traits at the 365 days (yearling) and 550 days (postyearling) of age. The variance components were estimated by the restricted maximum likelihood method. In the analysis of variance a model that included the fixed effects of sex, year and season and herd of birth, and the age of dam as covariant and the random effect of sire within the herd, was used. The fixed effects of classification were significant for all evaluated traits. Least square means were $186.16 \pm 2.74 \mathrm{~kg}$ and $244.06 \pm 5.23 \mathrm{~kg}$, for yearling and post-yearling weight respectively. Age of dam at calving influenced only the yearling weights, with estimated maximum weight of $187.29 \mathrm{~kg}$ and ages around nine years. Heritability estimates were $.56 \pm .09$ and $.64 \pm .12$, for yearling and post-yearling weight, respectively.
\end{abstract}

Key Words: corporal weight, heritability, genetic correlation

\section{Introdução}

A raça Nelore compõe quase $70 \%$ do rebanho zebuíno do Brasil, apresentando grandes diferenças no potencial do crescimento entre as várias regiões do país e também entre os vários sistemas de criação (ELER et al., 1995). Essa grande variabilidade constitui-se em base eficaz para melhorar geneticamente a produção de carne, principalmente no Estado do Nordeste, onde as limitações em função do clima e dos poucos trabalhos de pesquisa desenvolvidos nessa área dificultam o estabelecimento de programas de melhoramento.

As estimativas dos parâmetros genéticos fornecem informações importantes sobre a natureza genética das diferentes características e são necessárias para predizer as respostas diretas e correlacionadas da seleção, formular índices e escolher os métodos de seleção mais adequados, enquanto o conhecimento das fontes de variação não-genética permite identificar os fatores ambientais que causam variações nas produções, possibilitando que se evidenciem as diferenças devidas aos fatores hereditários, facilitando, dessa forma, a escolha dos indivíduos geneticamente superiores.

Entre os vários efeitos que influenciam o crescimento pós-desmame, ano e estação de nascimento têm importância particular, já que as condições climáticas podem ser diferentes durante os vários anos, o que significa também diferentes condições alimenta-

\footnotetext{
${ }^{1}$ Parte da dissertação de Mestrado apresentada à UFC - Fortaleza - CE pelo primeiro autor. Apoio financeiro: CAPES e CNPq

2 Estudante de Pós-Graduação em Zootecnia da Universidade Federal do Ceará.

3 Professor Adjunto, Departamento de Zootecnia, CCA/UFC. e-mail: martins@ufc.br

${ }^{4}$ Professor Titular, Departamento de Ciências Zootécnicas da Universidade de Firenze - Itália.

5 Doutor, Departamento de Ciências Zootécnicas da Universidade de Firenze - Itália. e-mail: giorgetti@zoot.agr.unifi.it
} 
res. Essa situação é muito comum no Nordeste do Brasil, onde a pecuária de corte caracteriza-se como exploração extensiva. MILAGRES et al. (1993), analisando 536 pesos aos 365 dias de idade de bezerros da raça Nelore criados Nordeste do Brasil, verificaram efeito significativo do ano de nascimento, do sexo, do tipo de manejo e do touro, não encontrando efeitos significativos de estação de nascimento e idade da mãe ao parto. OLIVEIRA e LÔBO (1992) estudaram os fatores ambientais relacionados com $\mathrm{o}$ peso aos 18 meses em bovinos Guzerá, relatando que houve influência significativa de sexo, estação e ano de nascimento. FIGUEIREDO et al. (1978), ainda na raça Nelore, estudando os pesos aos 365 e 550 dias de idade, encontraram significância para os efeitos de sexo $(208,1 \mathrm{~kg}$ e $263,8 \mathrm{~kg}$ para os machose $183,9 \mathrm{~kg}$ e $228,1 \mathrm{~kg}$ para as fêmeas) e da estação de nascimento, tendo os animais nascidos na época da seca sido mais pesados que os nascidos na época da chuva, (205,6 kg vs $190,1 \mathrm{~kg} ; 258,2 \mathrm{~kg}$ vs $239,1 \mathrm{~kg}$ ). MACHADO et al. (1997) estudaram a influência de fazenda, sexo, ano e época de nascimento, reprodutor, classe de regime alimentar e idade da vaca ao parto em animais Nelore nascidos entre 1976-1994 em 44 fazendas vinculadas a Associação Brasileiras Criadores Zebu, (ABCZ). As médias dos pesos aos 365 dias e aos 550 dias de idade, segundo o sexo do animal, foram de $240,7 \pm 2,5$ e $321,7 \pm 4,4 \mathrm{~kg}$ para os machos e 217,2 $\pm 2,5$ e 283,7 $\pm 4,4 \mathrm{~kg}$ para as fêmeas, respectivamente. No caso dos pesos aos 550 dias, não houve efeito significativo da época de nascimento. MARTINS FILHO et al. (1997) estudando, no Estado do Ceará, 848 pesos aos 365 dias e 437 pesos aos 550 dias de animais Nelore, encontraram médias de 165,12 $\pm 24,58 \mathrm{~kg}$ e $214,32 \pm 23,86 \mathrm{~kg}$, respectivamente. No mesmo trabalho, para 732 dados de ganhos em peso até os 365 dias e 321 ganhos em pesos até os 550 dias, esses autores encontraram média de $0,310 \pm 0,13 \mathrm{~kg}$ e $0,260 \pm 0,10 \mathrm{~kg}$, respectivamente.

MERCADANTE et al. (1995), em trabalho de revisão para as características de crescimento na espécie zebuina, encontraram os seguintes intervalos de herdabilidade: 0,05 a 0,58 para peso aos 205 dias, 0,12 e 0,93 para peso aos 365 dias de idade e 0,09 e 0,79 para peso aos 550 dias. Estimativas de herdabilidade obtidas para peso a 1 ano de idade em gado Nelore mostram variação de 0,20 a 0,46 , estando a média por volta de 0,30 (MILAGRES et al. 1993).

No presente estudo foram analisadas as influências de ano e estação de nascimento, sexo, fazenda e idade da mãe sobre o desempenho ponderal de bezer- ros Nelores, dos 365 aos 550 dias de idade, criados nos Estados do Ceará e do Piauí, e foram estimados os valores de herdabilidades para as duas características.

\section{Material e Métodos}

Os dados utilizados no presente estudo referemse a animais da raça Nelore incluídos no sistema de Controle de Desenvolvimento Ponderal (CDP), realizado pela Associação Brasileira de Criadores de Zebu (ABCZ), coletados durante 19 anos (19761994), provenientes de 11 fazendas distribuídas nos Estados do Ceará e do Piauí, e são resultado de pesagens trimestrais até os 18 meses de idade, efetuadas por técnicos da $\mathrm{ABCZ}$.

O arquivo geral dos dados continha 5252 observações, relativas a 2598 bezerros e 2654 bezerras, filhos de 167 touros e 2896 vacas, criados em regime de pasto onde receberam suplementação mineral.

Para efeito da análise dos dados e de acordo com a distribuição das chuvas durante o ano na região, os meses do ano foram agrupados em janeiro, fevereiro, março, abril, maio e junho (estação chuvosa $=1$ ) e julho, agosto, setembro, outubro, novembro e dezembro (estação seca $=2$ ).

As precipitações totais apresentaram distribuição irregular em relação aos anos, com valor máximo de $1840 \mathrm{~mm} /$ ano e mínimo de $479 \mathrm{~mm} / \mathrm{ano}$, no ano de 1985 e de 1983, respectivamente, e também em relação às duas diferentes estações: a estação chuvosa, que apresentou o maior valor foi a do ano de 1985 (1558 mm) e a estação seca, que apresentou as precipitações menores foi a do ano de 1983 .

Do arquivo geral foram utilizadas somente as informações relativas aos pesos a 1 ano e a um ano e meio de idade, eliminando-se também os reprodutores que tinham menos de seis filhos. Todos os pesos foram ajustados às idades-padrão de 365 e 550 dias, segundo fórmulas descritas por LÔBO (1992).

Para a característica peso aos 365 dias (P365) obtiveram-se 2004 observações, enquanto para o peso aos 550 dias (P550) as observações disponíveis foram 1077. A idade da mãe ao parto foi calculada em dias e utilizada nos modelos como covariável linear e quadrática.

As análises foram feitas no Laboratório de Informática do Departamento de Zootecnia-CCA da Universidade Federal do Ceará e no Departamento de Zootecnia da Faculdade de Agronomia da Universidade de Firenze Itália, utilizando-se os programas SAS (SAS, 1996) e MTDFREML (BOLDMAN et al., 1993).

O modelo matemático geral utilizado no procedi- 
470 Rev. bras. zootec.

mento GLM do programa SAS (SAS, 1996) foi:

$Y_{\text {jilkmno }}=\mu+F_{i}+R_{i j}+A_{k}+E_{l}+S_{m}+I_{n}+e_{i j k l m n o}$ em que

$Y_{\text {jilkmno }}=$ peso aos 365 e 550 dias de idade do $o$ ésimo filho do reprodutor $j$ dentro da fazenda $i$, nascido na estação $l$ do ano $k$, do sexo $m$ e da mãe com idade ao parto $n$;

$\mu=$ média geral para as características estudadas;

$F_{i}=$ efeito fixo da fazenda;

$R_{i j}=$ efeito aleatório do reprodutor $j$ dentro da fazenda $i$;

$A_{k}=$ efeito do ano do nascimento $k$;

$E_{l}=$ efeito da estação $l$;

$S_{m}=$ efeito do sexo da cria $m$;

$I_{n}=$ idade da vaca ao parto $n$, covariável; e

$e_{\text {jilkmno }}=$ erro aleatório, normal, independentemente distribuído com média zero e variância $\sigma^{2}$.

As estimativas dos componentes de (co)variância foram obtidos pelo método da Máxima Verossimilhança Restrita não Derivativa - DFREML, utilizando-se o programa MTDFREML (BOLDMAN et al., 1993). Uma vez que não houve informações do parentesco, foi utilizado um modelo de touro (Sire Model), assumindo que não havia reprodutores parentes.

Usando uma notação matricial o modelo de touro resulta ser:

$$
Y=X \beta+Z u+e,
$$

em que $Y$ é um vetor para as $n$ observações (os n pesos aos 365 dias, por exemplo); $\beta$, vetor de $p$ efeitos fixos (fazenda, ano e estação de nascimento, sexo e idade da mãe); $X$, matriz de incidência da ordem $n x$ $p$, que associa cada observação a cada efeito fixo; $u$, vetor dos efeitos genéticos aleatórios; $Z$, matriz da ordem $n \times t$ que associa cada touro às observações de sua progênie; e $e$, vetor dos efeitos residuais.

Os componentes de (co)variância obtidos foram utilizados para estimar as herdabilidades $\left(\mathrm{h}^{2}\right)$ para as características estudadas, utilizando a correlação intra classe de meio-irmãos paternos. Os errospadrão das estimativas foram calculados de acordo com a fórmula proposta por SWIGER (1964).

\section{Resultados e Discussão}

\section{Efeitos não-genéticos}

$\mathrm{Na}$ Tabela 1 é apresentado o resumo das análises de variância relativas aos pesos aos 365 dias e aos 550 dias de idade.

Todas as fontes de variação consideradas exerceram efeito significativo sobre o peso aos 365 dias $(\mathrm{P}<0,001)$, enquanto os efeitos linear e quadrático da covariável idade da mãe foram significativos a $5 \%$.

No caso do peso aos 550 dias, o efeito de touro, fazenda, ano de nascimento e sexo do bezerro foram altamente significativos $(\mathrm{p}<0,001)$, enquanto a estação de nascimento foi significativa a $5 \%$. Tanto o

Tabela 1 - Quadrado médio da análise de variância para os pesos aos 365 (P365) e 500 (P550) dias de idadde

Table 1 - Mean square of the analysis of variance for weights at 365 (P365) and 550 (P550) days of age

\begin{tabular}{|c|c|c|c|}
\hline $\begin{array}{l}\text { Fonte de variação } \\
\text { Source of variation }\end{array}$ & $\begin{array}{l}\mathrm{gl} \\
d f\end{array}$ & Qua & $\begin{array}{l}\text { do médio } \\
\text { square }\end{array}$ \\
\hline & & P365 & P550 \\
\hline Touro dentro de fazenda & $110(94)^{1}$ & $3784,28^{* *}$ & $6295,95 * *$ \\
\hline Sire within farm & & & \\
\hline Sexo & 1 & $65045,96 * *$ & $190565,93 * *$ \\
\hline $\operatorname{Sex}$ & & & \\
\hline Ano de nascimento & 18 & $12868,36^{* *}$ & $15218,13 * *$ \\
\hline Year of birth & & & \\
\hline Estação de nascimento & 1 & $30428,56 * *$ & $12804,63^{*}$ \\
\hline Season of birth & & & \\
\hline Rebanho & 10 & $53014,92 * *$ & $78358,04 * *$ \\
\hline Herd & & & \\
\hline Idade da vaca ao parto, linear & 1 & $5535,06^{*}$ & 271,02 \\
\hline $\begin{array}{l}\text { Cow age at calving, linear } \\
\text { Idade da vaca ao parto, quadrático }\end{array}$ & 1 & $6872,62 *$ & 485,84 \\
\hline $\begin{array}{l}\text { Cow age at calving, quadratic } \\
\text { Erro }\end{array}$ & $1861(952)^{1}$ & 1448,47 & 2769,93 \\
\hline $\begin{array}{l}\text { Error } \\
\mathrm{R}^{2}\end{array}$ & & 0,53 & 0,62 \\
\hline
\end{tabular}


efeito linear quanto o efeito quadrático da idade da mãe não foram significativos.

A média ajustada para o peso aos 365 dias foi de $186,16 \pm 2,74 \mathrm{~kg}$ e para o peso aos 550 dias, de 244,06 $\pm 5,23 \mathrm{~kg}$. Em ambos os casos, os valores obtidos são menores que os encontrados na mesma raça por OLIVEIRA FILHO e DUARTE (1987), MARIANTE et al. (1978), ELER et al. (1989), BARCELOS e LOBATO (1992), OLIVEIRA e LÔBO (1992), MILAGRES et al. (1993), AMARAL et al. (1986), ELER et al. (1991), FIGUEIREDO et al. (1978), MAGNABOSCO et al. (1996), MACHADO et al. (1997). No entanto, foram superiores ao encontrado por MARTINS FILHO et al. (1997), proveniente de animais criados no Estado do Ceará, e muito próximos aos valores achados por CUBAS et al. (1996) no Paraná. Os valores obtidos no presente estudo são inferiores a maioria dos valores encontrados na bibliografia em razão de os trabalhos revisados serem referentes a animais criados nas regiões do Centro-Sul do País, onde, como conseqüência das melhores condições climáticas, há maior disponibilidade de alimento durante o ano todo. Os rebanhos estudados encontram-se situados na região semi-àrida do País, onde as condições adversas de clima e solo, aliadas às deficientes práticas de manejo geralmente adotadas, podem ter contribuído para essa inferioridade.

Os machos foram mais pesados que as fêmeas nas duas idades consideradas, confirmando o melhor desempenho ponderal do sexo masculino.

$\mathrm{O}$ ano de nascimento exerceu efeito significativo tanto sobre o peso aos 365 dias como sobre o peso aos 550 dias de idade (Tabela 1). Os mesmos resultados foram obtidos por todos os autores revisados, à exceção de FIGUEREIDO et al. (1978), os quais não verificaram a influência desse efeito. $\mathrm{O}$ autor concluiu que não houve grande mudança com respeito ao manejo e à alimentação e que as condições climáticas médias foram semelhantes durante os anos em que os dados foram coletados. No presente estudo, ao contrário, as variações no manejo, na alimentação e nas condições climáticas foram as causas prováveis das diferenças entre os vários anos estudados.

No caso do peso aos 365 dias de idade, os animais mais pesados nasceram no ano de 1982 $(227,70 \pm 10,97 \mathrm{~kg})$ e os mais leves, no ano de 1980 $(162,03 \pm 9,39 \mathrm{~kg})$, enquanto para o peso aos 550 dias o peso máximo foi alcançado pelos bezerros nascidos no ano de $1980(289,37 \pm 25,56 \mathrm{~kg})$ e o menor, no ano de $1978(175,40 \pm 17,40 \mathrm{~kg})$.
A estação de nascimento, seca ou chuvosa, exerceu efeito significativo sobre ambas as características, embora com níveis de significância diferentes, $\mathrm{P}<0,001$ para o peso aos 365 e $\mathrm{P}<0,05$ para o peso aos 550 dias. O peso aos doze meses está ligado diretamente à disponibilidade de alimento, pois supõe-se que o bezerro não esteja mais mamando e, conseqüentemente, está sujeito às variações ocorridas na qualidade e na quantidade das forragens. Isso significa que, se o animal nasceu durante a estação chuvosa, ele será desmamado durante a estação seca, quando os pastos são escassos e, portanto, o seu crescimento até os doze meses ficará prejudicado. Ao contrário, nascendo no final da seca, o animal teria condição, uma vez desmamado, de conseguir alimentos suficientes para crescer rapidamente e sem dificuldades, pelo menos até um ano de idade.

A partir dessa idade (12 meses) pode ocorrer a influência da forma de manejo, uma vez que a maioria dos criadores, principalmente na estação seca, fornece aos animais suplementação alimentar em forma de volumoso e, por conseguinte, o animal acaba recebendo alimento suficiente para crescer, mesmo no período mais difícil do ano. Pode acontecer nessa faixa etária que o bezerro obtenha ganho em pesos maiores na estação seca que na estação chuvosa, em decorrência dessa suplementação alimentar. Essa hipótese aplica-se ao comportamento do peso aos 550 dias, pois os animais nascidos na época seca tiveram peso maior que os nascidos na época da chuva, 248 $\mathrm{kg}$ contra $239 \mathrm{~kg}$, respectivamente. Vários autores encontraram os mesmos resultados, entre eles: FIGUEREIDO et al. (1978), ROSA et al. (1986), ELER et al. (1989), LÔBO et al. (1993), MACHADO et al. (1997). Essas informações relativas ao desempenho do animal, em função da época do nascimento, são muito importantes para o criador que vende os seus animais a partir de 1 ano de idade.

Outra fonte de variação incluída no modelo que também se mostrou significativa $(\mathrm{P}<0,001)$ foi a fazenda onde os animais nasceram. Esse efeito, para os pesos pós-desmame, é conseqüência de diferenças climáticas, que se refletem na quantidade e qualidade dos alimentos disponíveis além do manejo e da composição genética de cada rebanho. OLIVEIRA FILHO e DUARTE (1987), ROSA et al. (1986), ELER et al. (1989), MILAGRES et al. (1993) e MACHADO et al. (1997) são alguns dos autores que obtiveram os mesmos resultados na raça Nelore.

A idade da mãe ao parto, quando inserida no modelo como covariável, foi significativa somente no 
472 Rev. bras. zootec.

caso do peso aos 365 dias $(\mathrm{P}<0,05)$, apresentando $b_{1}=0,006189$ e $b_{2}=-0,0000095$. Aos 365 dias de idade, supõe-se que o bezerro seja totalmente independente na sua alimentação, mas isso muitas vezes não acontece, principalmente nas condições de criação do Nordeste, onde os animais vivem a campo o ano todo. Assim, o desempenho do bezerro recebe ainda a influência do efeito materno e a idade de um ano pode ser considerada em algum caso como a idade do efetivo desmame. O efeito quadrático da regressão confirma o que foi encontrado por outros autores (CUNDIFF et al., 1966; TROVO e RAZOOK, 1995) - as vacas mais novas e as mais velhas foram as que desmamaram os bezerros mais leves. Desenvolvendo a equação, observa-se que o peso máximo foi de $187,69 \mathrm{~kg}$, ocorrendo para filhos de matrizes com idade em torno de 9 anos (3257 dias), como mostra a Figura 1. A idade elevada das matrizes é, provavelmente, conseqüência do tipo de manejo prevalecente na região, onde não existe estação de monta regular e as vacas entram em reprodução tardiamente, conseqüência de alimentação não adequada.

As estimativas de herdabilidade dos pesos aos 365 e 550 dias de idade foram $0,56 \pm 0,09$ e $0,64 \pm 0,12$, respectivamente. No caso do peso aos 365 dias, o valor obtido foi maior que todos os valores encontrados nos trabalhos revisados, cujo intervalo situou-se entre 0,17 e 0,45 . Nas condições de criação típicas do Nordeste, os criadores selecionam os animais mais em função das características anatômicas e raciais que em função do desempenho produtivo, o que não deve interferir, portanto, na variabilidade genética, que permanece elevada. Além disso, deve ser considerado que a maioria dos valores encontrados na bibliografia foi obtida pela metodologia dos quadrados mínimos, a qual pode ter subestimado os parâmetros genéticos. FREITAS e VENCOVSKY (1992), para a característica peso aos doze meses, obtiveram valores de herdabilidade iguais a $0,39 \pm$ 0,10 , utilizando o Método Henderson 3 e $0,43 \pm 0,14$ com o método REML.

No caso dos pesos aos 550 dias, o valor da estimativa de herdabilidade obtido $(0,64 \pm 0,12)$ situase no intervalo de 0,09 a 0,79 , encontrado por MERCADANTE et al. (1995). Os valores para herdabilidade para a característica encontrada na bibliografia consultada, para a raça Nelore, são menores que o obtido neste estudo, com a exceção dos achados de ROSA et al. (1986). Os resultados desta pesquisa confirmam a hipótese de existência de grande variabilidade genética nos rebanhos analisados,

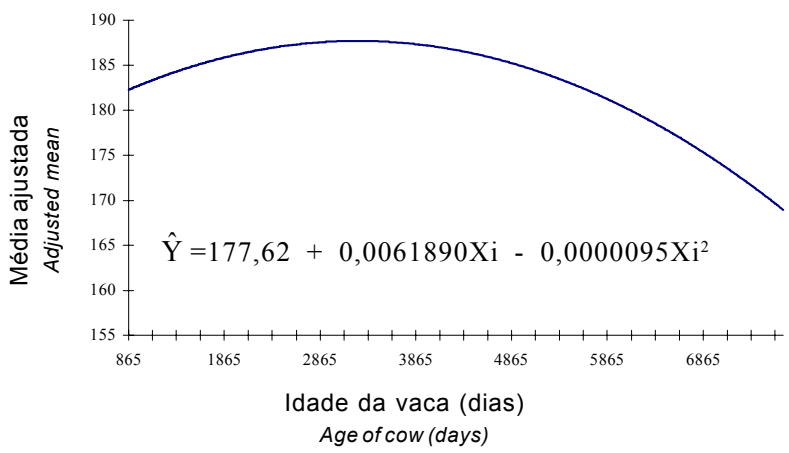

Figura 1 - Regressão do peso aos 365 dias em relação à idade das vacas.

Figure 1 - Regression of weight at 365 days on age of cow.

para as características estudadas.

\section{Conclusões}

O crescimento ponderal dos 365 até os 550 dias de idade foi influenciado pelo efeitos fixos de estação e ano de nascimento, sexo e fazenda. A estação de nascimento constitui fonte de variação importante, em que os animais nascidos na estação seca apresentam pesos maiores que os nascidos na estação chuvosa.

Os machos foram mais pesados que as fêmeas em todas as idades, com ganho de peso $30 \%$ mais elevado, dos 365 até os 550 dias.

A idade da mãe ao parto influenciou somente o peso aos 365 dias, com peso máximo de $187,29 \mathrm{~kg}$, a uma idade em torno de 9 anos.

As herdabilidades estimadas para as duas características foram de magnitude média a alta.

\section{Referências Bibliográficas}

AMARAL, C.O. LÔBO, R.B., DUARTE, F.A.M. et al. Coeficientes de herdabilidade e correlação genética entre características de crescimento em bovinos da raça Nelore. In: REUNIÃO ANUAL DA SOCIEDADE BRASILEIRA DE ZOOTECNIA, 23, 1986, Campo Grande-MS. Anais... Campo Grande-MS: SBZ, 1986. p. 309.

BARCELOS, J.O.J., LOBATO, J.F.P. 1992. Efeitos da época de nascimento no desenvolvimento de bezerros Hereford e suas cruzas. II-pesos ao desmame, ano e sobreano. R. Soc. Bras. Zootec., 21(1):150-157.

BOLDMAN, K.G., KRIESE, L.A., VAN VLECK, D.L. et al. 1993. A Manual for use of MTDFREML-A set of programs to obtain estimates of variances and covariances. ARS, USDA. 
CUBAS, A.C., PEROTTO, D., ABRAhÃO, J.J.S. et al. Desempenho ponderal de animais Nelore e cruzas com Nelore. II. Período Pós-desmama. In: REUNIÃO ANUAL DA SOCIEDADE BRASILEIRA DE ZOOTECNIA, 33, Fortaleza, 1996. Anais... Fortaleza: 1996. p 127-129

CUNDIFF, L.V., WILLHAM, R.L., PRATT, C.A. 1966. Effects of certain factors and their two-way interactions on weaning weigth in beef cattle. J. Anim. Sci., 25:972-982.

ELER, J.P., FERRAZ, J. B. S., LÔBO, R.B. et al. Influência de alguns fatores genéticos e de meio em pesos de bovinos da raça Nelore de um rebanho do Estado de São Paulo. In: REUNIÃO ANUAL DA SOCIEDADE BRASILEIRA DE ZOOTECNIA, 28, 1991, João Pessoa, PB. Anais... João Pessoa: SBZ, 1991, p.553.

ELER, J.P., LÔBO, R.B., ROSA, A.N. 1989. Influência de fatores genéticos e de meio em pesos de bovinos da raça Nelore criados no Estado de São Paulo. R. Soc. Bras. Zootec., 18(2):103-111.

ELER, J.P., VAN VLECK, L.D., FERRAZ, J.B.S. et al. 1995. Estimation of variance due to direct and maternal effects for growth traits of Nelore cattle. J. Anim. Sci., 73:3253-3258.

FIGUEIREDO, G.R., ALMEIDA, M., MILAGRES, J.C. et al. 1978. Estimativas de parâmetros genéticos e fenotípicos de pesos e ganhos de peso de animais Nelore após a desmama. R. Soc. Bras. Zootec., 7(2):287-302.

FREITAS, A.R., VENCOSKY, R. Métodos de estimação de variância e parâmetros afins de características de crescimento em bovinos. In: REUNIÃO ANUAL DA SOCIEDADE BRASILEIRA DE ZOOTECNIA, 29, 1992, Lavras, MG. Anais... Lavras: SBZ, 1992. p.119.

LÔBO, R.B. 1992.Programa de melhoramento genético da raça Nelore, 2.ed., Ribeirão Preto - SP: Universidade de São Paulo.

LÔBO, R.B., OLIVEIRA, H.N., MERCADANTE, M.E.Z. et al. Estimativa de herdabilidade para características de crescimento na raça Nelore. In: CONGRESSO NACIONAL DE GENÉTICA, 39, 1993, Caxambu, MG. Anais... São Paulo: SBG, 1993, p.390.

MACHADO, P.F.A., AQUINO, L.H., GONÇALVES, T.M. et al. Influência de fatores de meio sobre características produtivas de animais da raça Nelore. In: REUNIÃO ANUAL DA SOCIEDADE BRASILEIRA DE ZOOTECNIA, 34,1997, Juiz de Fora, MG. Anais... Juiz de Fora: SBZ, 1997 , p 211-213.

MAGNABOSCO, C.U., FAMUlA, T.R., LÔBO, R.B. et al. Estimativas de parâmetros genéticos e de ambiente de características de crescimento em bovinos da raça Nelore. In: REUNIÃO ANUAL DA SOCIEDADE BRASILEIRA DE ZOOTECNIA, 33, 1996, Fortaleza, CE. Anais... Fortaleza: SBZ, 1996. p.142-144.
MARIANTE, A., HARGROVE, D. D., KOGER, M. et al. 1978. Factors affecting growth of Nellore cattle in central Brazil. $J$. Anim. Sci., 46:6 (suplemento).

MARTINS FILHO, R., LOBO, R. N. B., LIMA, F. A. M. et al. Parâmetros genéticos e fenotípicos de pesos e ganhos em pesos de bovinos zebus no Estado do Ceará. In: REUNIÃO ANUAL DA SOCIEDADE BRASILEIRA DE ZOOTECNIA, 34, 1997, Juiz de Fora, MG. Anais... Juiz de Fora: SBZ, 1997. p.248-250.

MERCADANTE, M. E. Z., LÔBO, R. B., BORJAS, A. DE LOS R. 1995. Parâmetros genéticos para características de crecimento en zebuínos de carne. Arch. Latin. Prod. Anim., 3(1):45-89.

MILAGRES, J.C., ARAÚJO, C.R., TEIXEIRA, N.M. et al. 1993. Influências de meio e herança sobre os pesos ao nascer, aos 205 e aos 365 dias de idade de animais Nelore criados no Nordeste do Brasil. R. Soc. Bras. Zootec., 22(3):455-465.

OLIVEIRA, J. A., LÔBO, R. B. 1992. Fatores ambientes e genéticos relacionados com o peso aos dezoito meses e ganho diário em bovinos Guzerá. R. Soc. Bras. Zootec., 21(4):629-636.

OLIVEIRA-FILHO, E.B., DUARTE, F. A.M. 1987. Peso e ganho em peso de bovinos Nelore criados no Estado de São Paulo, da desmama aos 365 dias. Ars Veterinária, 3(1):105-112.

ROSA, A.N., SILVA, L.O.C., NOBRE, P.R.C. 1986. Avaliação do desempenho de animais Nelore em controle de desenvolvimento ponderal no Estado do Mato Grosso do Sul, Brasil. R. Soc. Bras. Zootec., 15(6):515-532.

STATISTICAL ANALYSIS SYSTEM. 1996. User's guide: Statistics. Version 6.11, NC; SAS Institute.

SWIGER, L.A., HARVEY, W.R., EVERSON, D.O. et al. 1964. The variance of intraclass correlation involving groups with one observation. Biometrics, 20:818-826.

TROVO, J.B., RAZOOK, A.G. 1995. Fundamentos da Avaliação Genética. In: I CURSO SOBRE AVALIAÇÃO GENÉTICA EM BOVINOS DE CORTE, 1995, Ribeirão Preto, p.1-19.
Recebido em: 30/04/98 Aceito em: 05/11/98 